\title{
Study on Vehicle Location in Road Traffic Accident Based on Surveillance Video and Vanishing Point
}

\author{
Aowen Duan ${ }^{1,}$ a, Wenjun Liu ${ }^{1, b}$, Hongchun Jia ${ }^{2, ~ c}$, Zhiyong Yin ${ }^{1, d, ~ * ~}$ \\ ${ }^{1}$ Institute for Traffic Medicine, Department 4th, Institute of Surgery Research, Daping Hospital, Third \\ Military Medical University, Chongqing, 400042, China; \\ ${ }^{2}$ College of Vehicle Engineering, Chongqing institute of technology, Chongqing, 400054, China. \\ aduanaowen@foxmail.com, b303924823@qq.com, c351042178@qq.com, d147210@qq.com
}

Keywords: surveillance video, vanishing point, road traffic accident, distance measurement.

\begin{abstract}
To solve the problem that it is difficult to obtain the accurate locations of target objects directly by the surveillance video in Road Traffic Accidents, this paper presents a method of measuring the relative position of vehicles on the road surface by using vanishing point principle to mesh the surveillance video frames. In a road traffic accident with surveillance video, this method was applied to analyze the dynamic positional relationship between vehicles and marking lines on the road surface. The accurate moving tracks of 2 vehicles involved were obtained and the measurement accuracy was verified. The result shows that the measurement errors are less than $3 \%$, proving that this method has a guiding effect on obtaining the accurate locations of vehicles in Road Traffic Accidents. It can be applied to traffic accidents with surveillance video to determine the accurate locations of target objects in the process, thus providing necessary data for subsequent accident reconstruction and injury reconstruction.
\end{abstract}

\section{Introduction}

With the deepening of urbanization in China and the general improvement of citizen's traffic safety awareness, the government has invested a large amount of money in the installation of road surveillance cameras. Meanwhile, other high-definition video recording equipment such as driving recorder is being widely used, making more and more road traffic accidents attached with high-quality video data [1]. According to the statistics of Chongqing Bayi Traffic Accident Judicial Appraisal Center, the percentage of accidents with video recording has obviously increased in the past four years (2014-2017), both exceeding 30\%. In 2017, it reached 65\% and showing a feature of gradual acceleration year by year (fig.1). Therefore, the determination of the traffic accident process through video data has developed into a new method of accident analysis, which can not only help traffic regulators reconstruct the accident so as to find the causes, but also provide accurate locations of the vehicles involved to traffic safety researchers for further study.

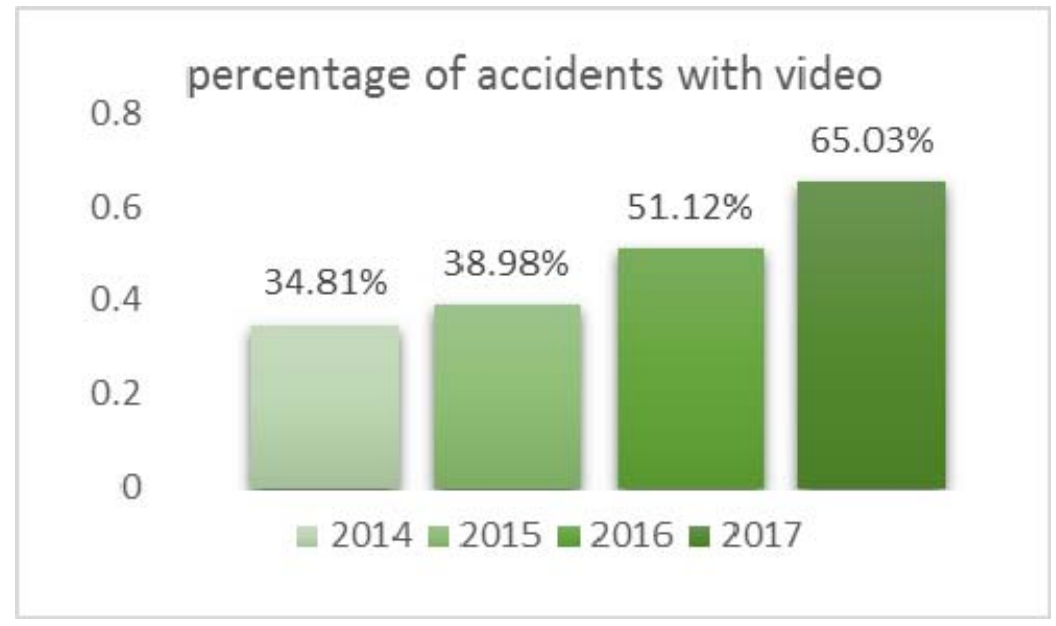

Fig. 1 Accident cases with video in the past four years 
Usually, due to the influence of shooting angle, distance and light, the quantitative positional relationship between vehicles/pedestrians and the road surface is hard to obtain directly through observing the video, which is essential for the traffic accident reconstruction and injury reconstruction. However, with the development of visual measurement technology, it proved that the distance between objects in video can be measured through photogrammetry for single images with high accuracy [2]. Therefore, the multi-frame images extracted from the surveillance video can be processed and analyzed to solve the problem of distance measurement and specify the vehicle/pedestrian locations during the whole period of an accident [3].

\section{Imaging Principle Of Surveillance Camera}

\subsection{Pinhole Camera Model.}

The camera's imaging principle can be usually expressed by a pinhole model (Fig. 2), in which $\left(X_{w}, Y_{w}, Z_{w}\right)^{T}$ represents three-dimensional world coordinates of point $\mathrm{P},\left(X_{c}, Y_{c}, Z_{c}\right)$ is the same point in the camera coordinate system. The camera coordinate system is defined as: the center is at $O_{c}$ (optical center), the $z_{c}$ axis coincides with the optical axis, the $x_{c}$ axis and the $y_{c}$ axis are parallel to the $\mathrm{X}$ axis and the $\mathrm{Y}$ axis respectively. The OXY plane is defined as the image plane, so the spatial point and the image plane constitute the ideal perspective correspondence. $F$ is the focal length of the ideal imaging system and $(u, v)$ represents the coordinates of frame illustrated by computer screen, whose unit is pixel and $\left(u_{0}, v_{0}\right)$ represents the center coordinate [4].

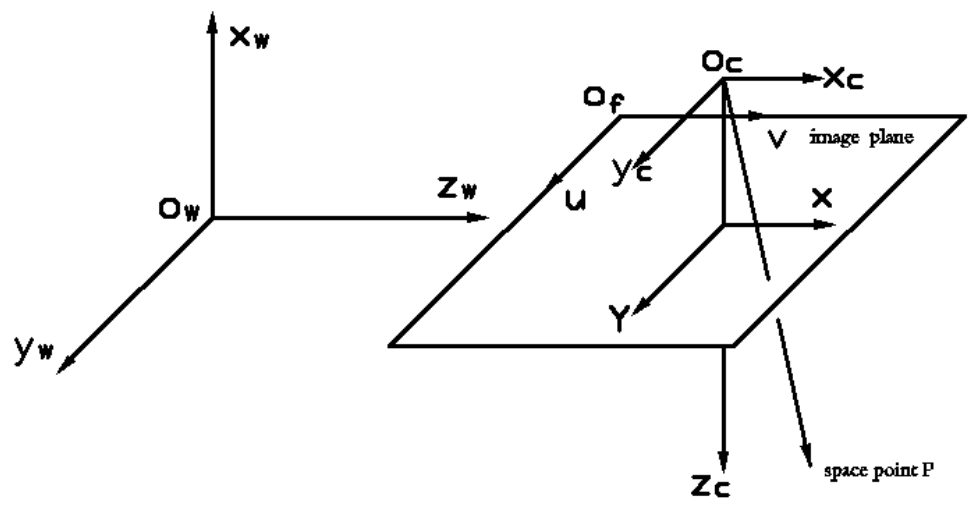

Fig. 2 Pinhole camera model

The projection relationship between the three-dimensional coordinates $\left(X_{w}, Y_{w}, Z_{w}\right)^{T}$ of the space point $\mathrm{P}$ and the pixel coordinates $(u, v)^{T}$ of its image plane can be expressed as:

$$
\alpha(\mu, \nu, 1)^{T}=M\left(X_{w}, Y_{w}, Z_{w}, 1\right)^{T}=K[R T]\left(X_{w}, Y_{w}, Z_{w}, 1\right)^{T}
$$

Where $\alpha$ is an arbitrary constant, $(\mu, v, 1)^{T}$ and $\left(X_{w}, Y_{w}, Z_{w}, 1\right)^{T}$ are the corresponding homogeneous coordinates, $M$ is a $3 \times 4$ projection matrix, $K$ is the camera's internal parameter matrix, $R$ and $T$ represent the rotation matrix and translation matrix of the camera coordinate system in the world coordinate system respectively, $\left(u_{0}, v_{0}\right)$ is the coordinates of the main point, $f_{u}$ and $f_{v}$ are the focal distances in the $u$ and $v$ directions respectively(in pixels), and $s$ is the skewness coefficient describing the two axes of the image plane.

\subsection{Vanishing Point.}

The parallel lines never intersect each other in Euclidean space. But in projective geometry, parallel lines intersect at infinity and the projection point of the intersection in the image plane is called the vanishing point. It has an important property: the line connecting the vanishing point and the camera's optical center $\mathrm{O}$ is parallel to any space parallel line forming the vanishing point. In most cases valuable information in road traffic accidents exists on the road surface and only lane marks are 
obvious parallel lines on the ground. With this property of vanishing point, reference lines which do not exist on the ground can be marked in the form of a grid to establish the coordinate system of the road surface, thus help locating the target objects accurately in the accident $[5,6]$. This method is applied to an accident with surveillance video to analyze the locational relationship of the vehicles on the road ground in this article.

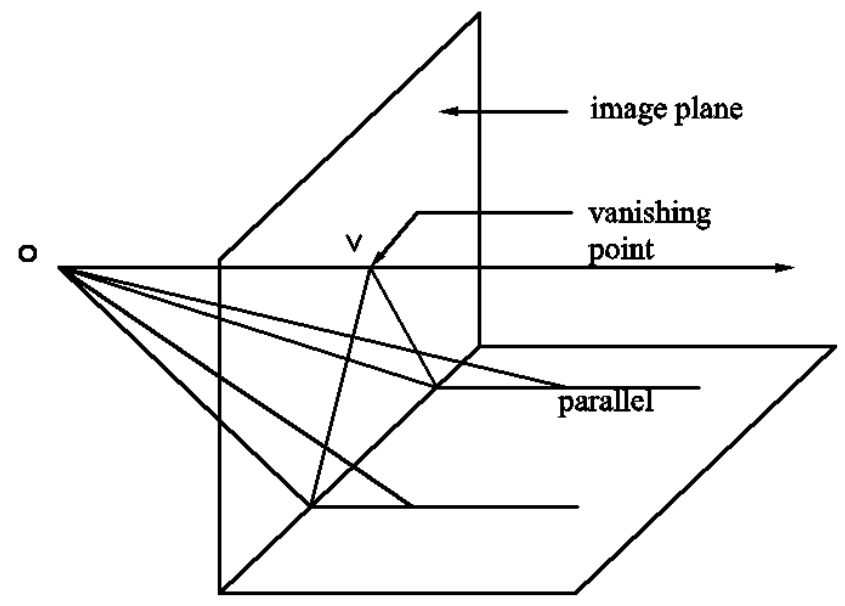

Fig. 3 Vanishing point property

\section{Accident Case Analysis}

\subsection{Brief Description of the Accident.}

One day in 2016, an unlicensed motorcycle and a freight truck were involved in a traffic accident when driving in an urban right-turn junction. A surveillance video saved by a camera near the scene revealed that the motorcycle was going straight and the truck was turning to the right and then the accident happened. In order to determine their driving status, the two vehicles' moving track needs to be located accurately. The scene of the accident and surveillance video frame are shown in Fig. 4.

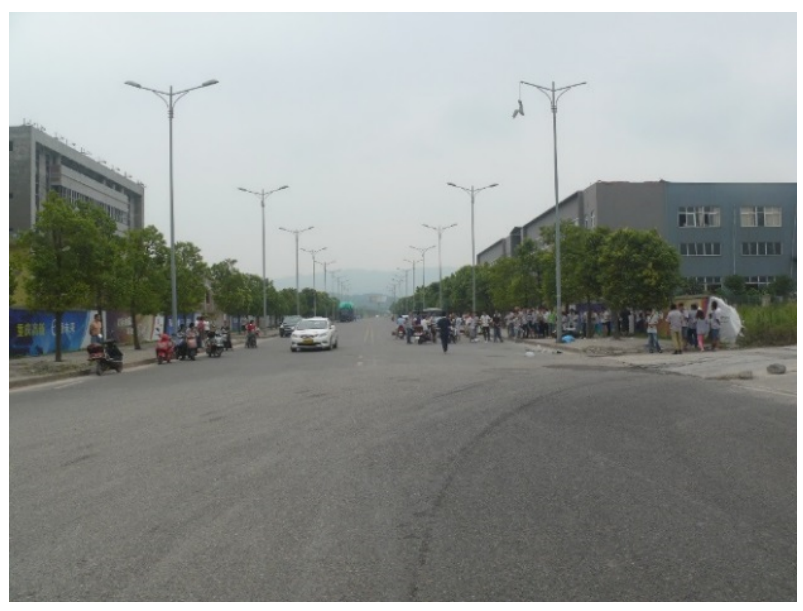

(a) Scene circumstance

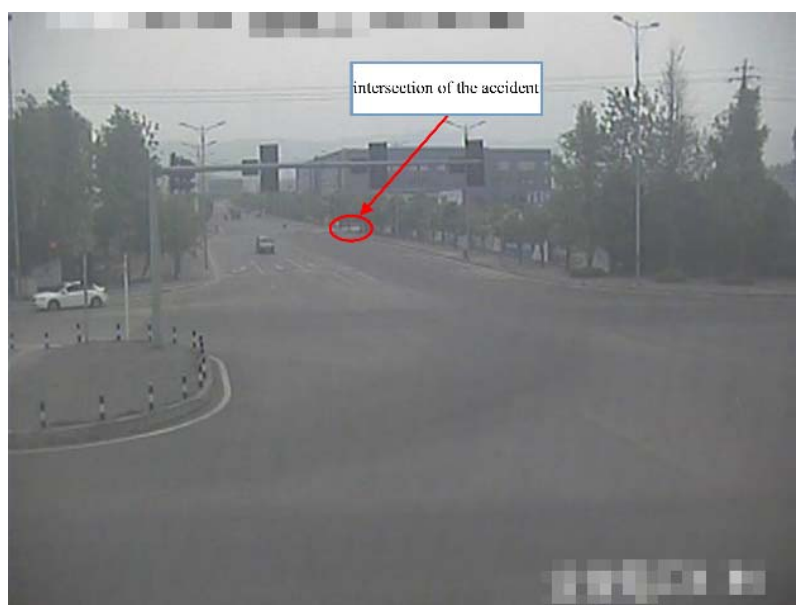

(b) Surveillance video frame

Fig. 4 Accident Scene

\subsection{Reconstruction of the Accident Scene.}

Multiple lengths of different road marking of the intersection were measured and recorded with a tape and the data will be used to evaluate video image measurement accuracy. At the same time, UAV(Unmanned Aerial Vehicle) was used to capture a wide range of road sections where the accident happened and it is clear that the road is basically straight with unchanged width. According to measurement, the distance between the lamppost and the stop line is 96 meters, the distance between the extension line of the road curb and the left marking line is 15.5 meters, the distance from the left lane to the left marking line ranges from 7.8 meters to 11.4 meters, the distance from the right 
lane to the left marking line ranges from 11.4 meters to 15.2 meters. The width of the intersection is 9.2 meters, the distance from the intersection to the lamppost ranges from 7.2 meters to 16.4 meters as shown in Fig. 5.

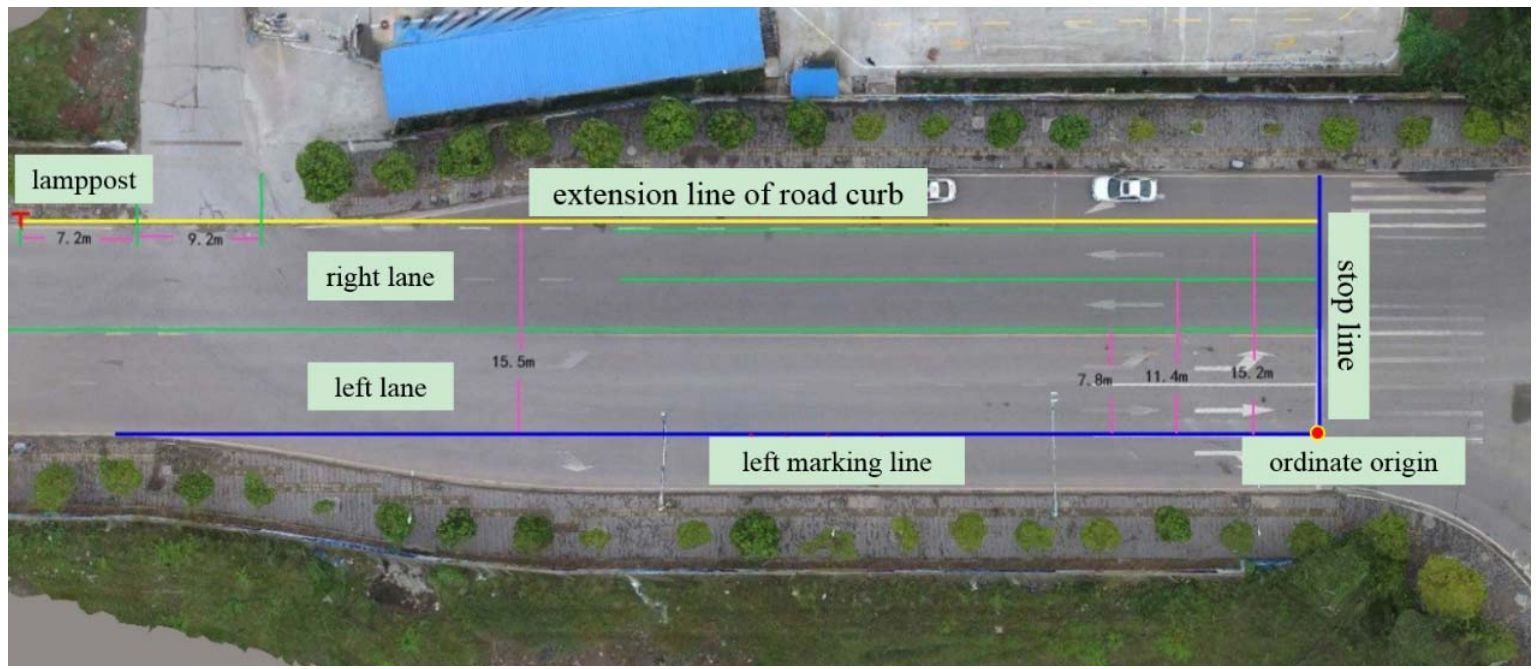

\subsection{Road Grid Coordinate System.}

Fig. 5 Top view of the accident scene

\subsubsection{Establishing the Grid Coordinate}

Based on a frame of the surveillance video, road surface grid coordinate system was established according to vanishing point principle in Photoshop CC software. As can be seen from Fig. 6, the blue plane represents the road surface where the distances between adjacent parallel lines in the $\mathrm{x}$ and $\mathrm{y}$ directions are equal. One group of the measured values of road marking are used to calibrate the vertical and horizontal criterion of the grid coordinate system to obtain the measurable grid plane.

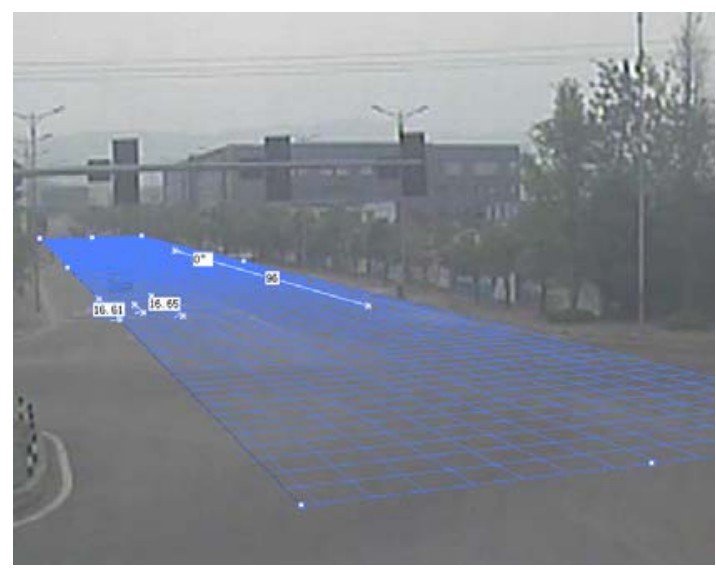

(a)Vertical calibration

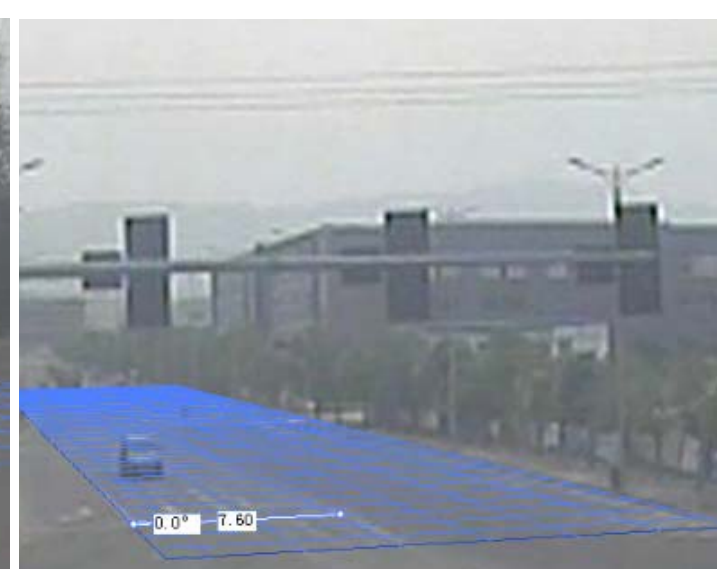

(b)Horizontal calibration

Fig. 6 Measurable grid coordinate

\subsubsection{Measurement Accuracy Validation}

The length of three groups of ground markings was recorded in the accident scene and the corresponding length was measured by the road grid plane as well. Then the actual value of the road marking was compared with the measured value and the results are shown in Table 1.

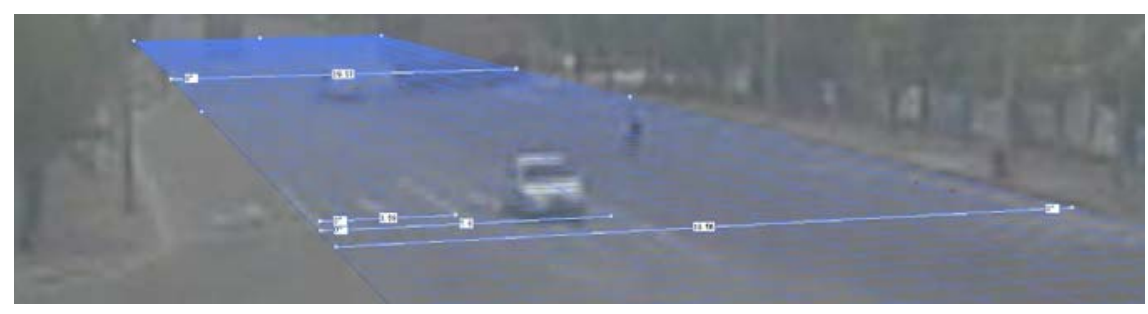

(a)Horizontal validation 


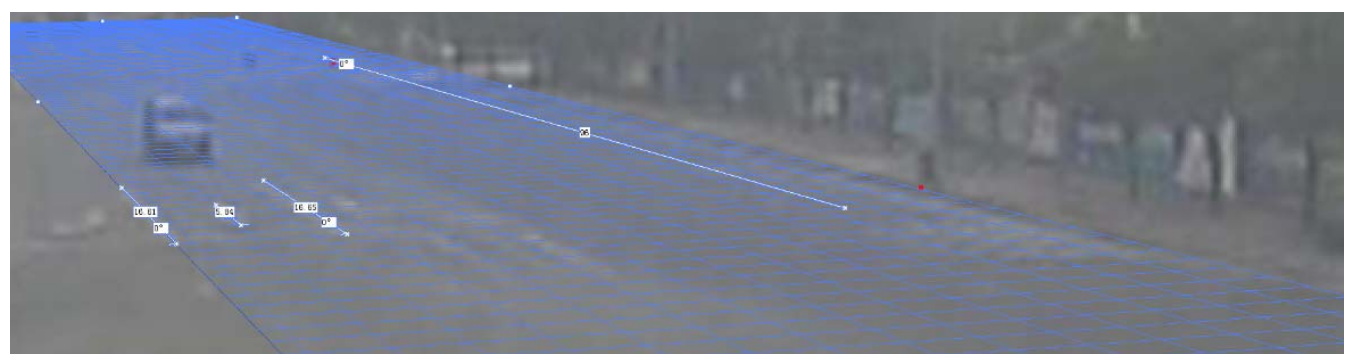

(b)Vertical validation

Fig. 7 Marking measurement and validation

The accident scene is far away from the surveilling spot, making it difficult to identify the road marking precisely. Meanwhile, the video image resolution is low, resulting in a certain error of both the horizontal and vertical measurements. According to the results, the maximum error of horizontal measurement is $0.31 \%$, the maximum error of vertical measurement is $2.63 \%$. The error of both directions is less than $3 \%$ which is within acceptable limits. It's believed that the grid plane can be used to calculate the position of vehicles in the accident.

Table 1. Measurement error of grid plane

\begin{tabular}{ccccccccc}
\hline & \multicolumn{3}{c}{ Vertical direction } & & \multicolumn{3}{c}{ Horizontal direction } \\
\cline { 1 - 3 } \cline { 6 - 8 } Measured value(m) & 5.84 & 16.65 & 16.61 & & 19.57 & 19.56 & 3.59 \\
Actual value(m) & 5.94 & 17.10 & 17.05 & & 19.62 & 19.62 & 3.60 \\
Error & $1.68 \%$ & $2.63 \%$ & $2.58 \%$ & & $0.25 \%$ & $0.31 \%$ & $0.28 \%$ \\
Mean error & & $2.30 \%$ & & & & $0.28 \%$ & \\
\hline
\end{tabular}

\subsection{Motorcycle Driving Track Analysis.}

The motorcycle appears at 16:48:53 and images were captured frame by frame from the surveillance video. Taking the projection point on the ground of motorcycle tail as the marker point, the driving track including points in 15 frames from the time 16:48:53 to 16:49:03 was determined. The road grid coordinate system was applied to measure the motorcycle track points on the road, as shown in Fig. 8.

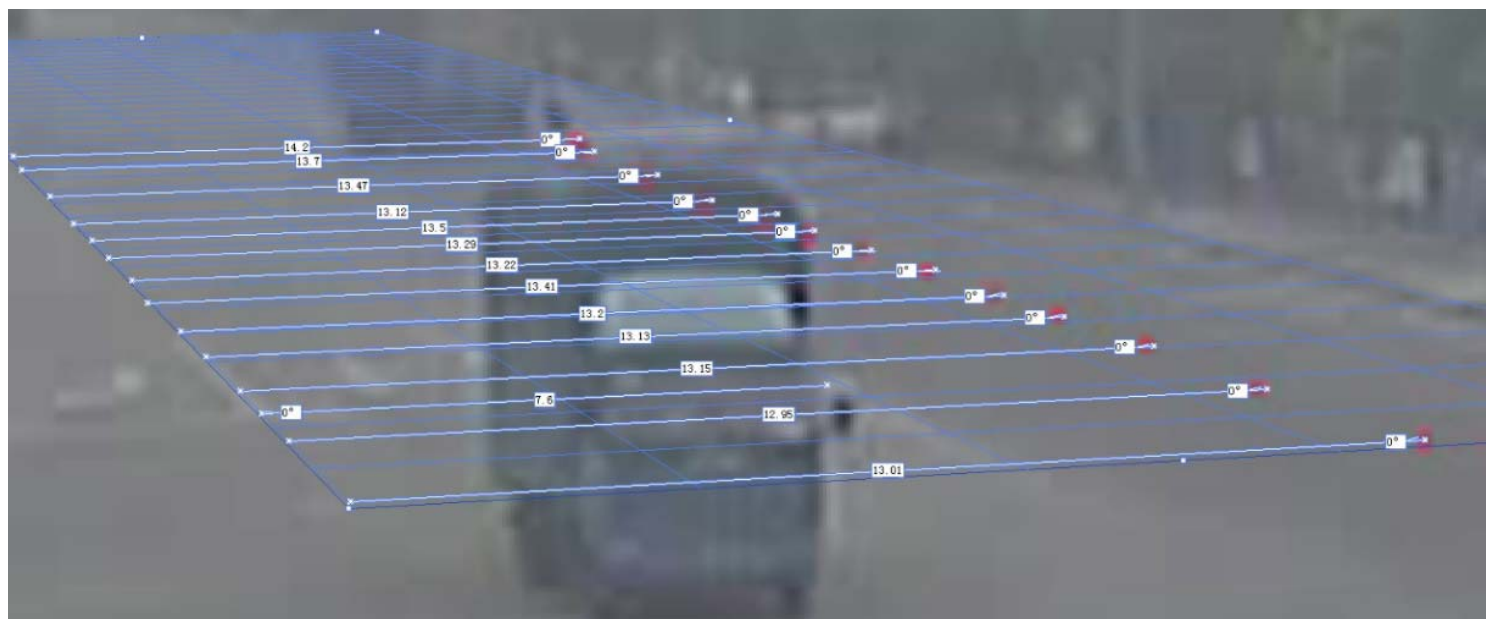

Fig. 8 Motorcycle track points

Taking the left standard line as the reference, the horizontal distance of each track point of motorcycle was measured as shown in Table 2. Based on the measured value, scatterplot of motorcycle moving track was made and the change of motorcycle driving path was analyzed accordingly.

Table 2. Horizontal distance of motorcycle track

\begin{tabular}{|c|c|c|c|c|c|c|c|c|c|c|c|c|c|}
\hline Point NO. & 1 & 2 & 3 & 4 & 5 & 6 & 7 & 8 & 9 & 10 & 11 & 12 & 13 \\
\hline Distance(m) & 13.01 & 12.95 & 13.15 & 13.13 & 13.2 & 13.41 & 13.22 & 13.29 & 13.5 & 13.12 & 13.47 & 13.7 & 14.2 \\
\hline
\end{tabular}


It can be seen from Fig. 9 that the distance of motorcycle movement track ranges from 12.95 meters to 14.2 meters. Taking the left standard line as the reference, the distance of the right lane in motorcycle driving direction ranges from 11.4 meters to 15.2 meters. Based on the data above, it can be inferred that the motorcycle driving track is in the right lane with an upward trend. That is to say, the motorcycle was moving in the right lane in its driving direction with a tendency to shift to the right before the accident.

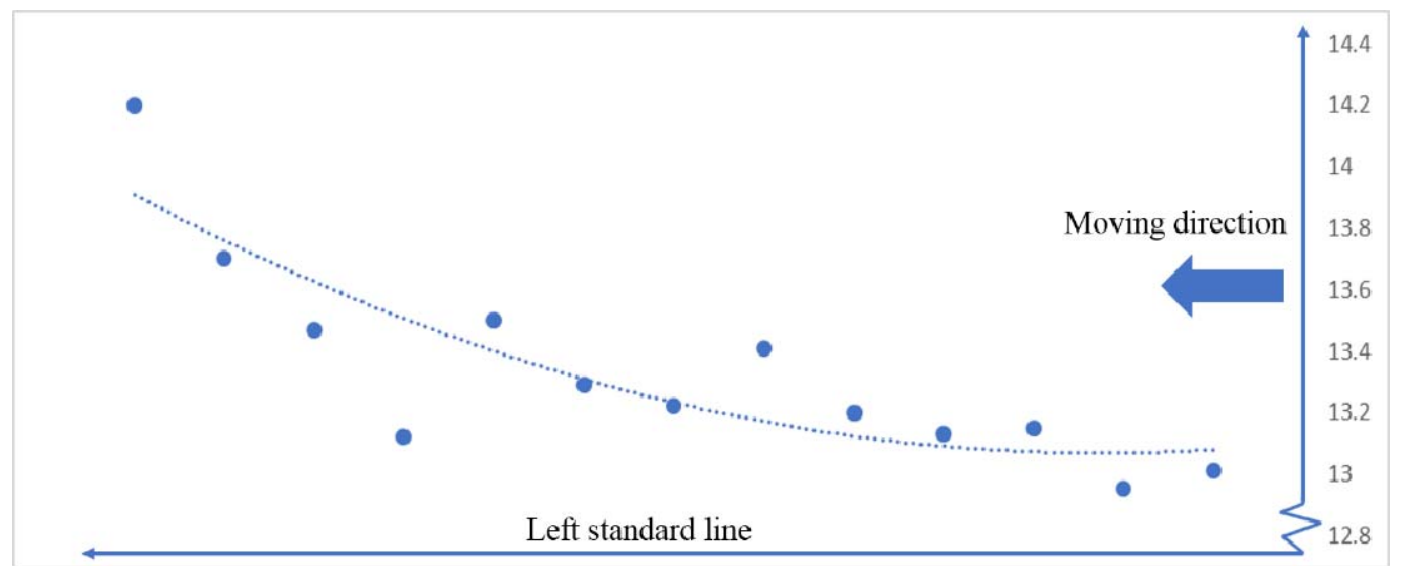

\subsection{Truck Driving Track Analysis.}

Fig. 9 Top view of motorcycle track point moving trend

The truck moves into the video screen at 16:48:34, enters the stop line at 16:48:47, and stops at 16:49:05. According to the video frames of this period of time, taking the left rear wheel of the truck and the right rear wheel as the reference targets, the moving track of the truck after entering the stopping line section was analyzed. As shown in Fig. 10, the red point indicates the projection point of the truck's left rear wheel on the ground, and the blue point indicates the projection point of the truck's right rear wheel on the ground, and the grid plane was used to measure the accurate position of these points.

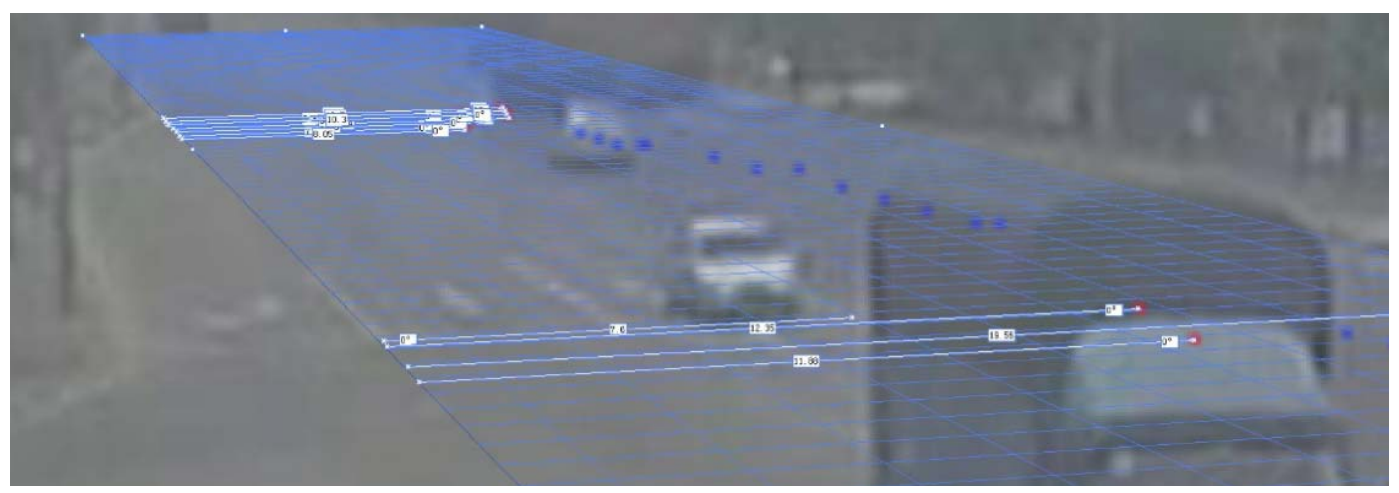

(a)Truck left rear wheel track point(red point)

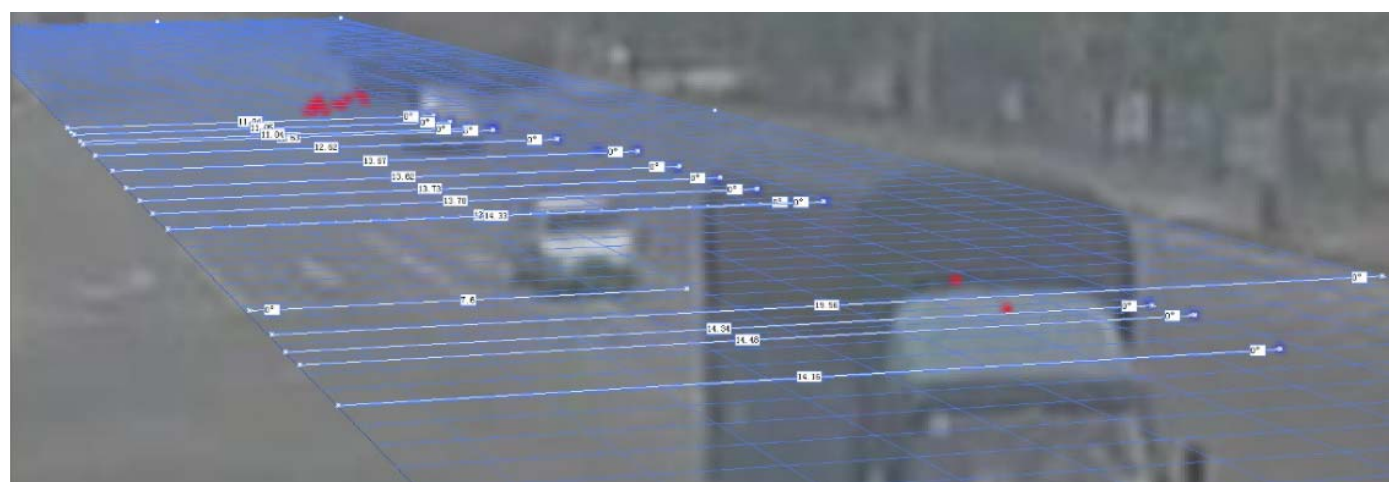

(b)Truck right rear track point(blue point)

Fig. 10 Truck moving track 
It is known that the width of the truck is 2.496 meters and taking the left standard line as the reference, the distance from the left rear wheel to the left standard line can be calculated after the position of the right rear wheel track point are measured which is shown on Fig.10 (b). Combined with the measured data in Fig. 10 (a), the distance from the left rear wheel to the left marking line of D1 to D26 is obtained (Table 3) and scatter plot is made accordingly (Fig. 11).

Table 3. Horizontal distance of left rear wheel track

\begin{tabular}{|c|c|c|c|c|c|c|c|c|c|c|c|c|c|}
\hline $\begin{array}{c}\text { Point NO. } \\
\text { Distance(m) }\end{array}$ & $\begin{array}{c}1 \\
11.66\end{array}$ & $\begin{array}{c}2 \\
11.98\end{array}$ & $\begin{array}{c}3 \\
11.84\end{array}$ & $\begin{array}{c}4 \\
11.88\end{array}$ & $\begin{array}{c}5 \\
12.35\end{array}$ & $\begin{array}{c}6 \\
11.83\end{array}$ & $\begin{array}{c}7 \\
11.38\end{array}$ & $\begin{array}{c}8 \\
11.28\end{array}$ & $\begin{array}{c}9 \\
11.23\end{array}$ & $\begin{array}{c}10 \\
11.12\end{array}$ & $\begin{array}{c}11 \\
11.17\end{array}$ & $\begin{array}{c}12 \\
10.12\end{array}$ & $\begin{array}{c}13 \\
9.13\end{array}$ \\
\hline Point NO. & 14 & 15 & 16 & 17 & 18 & 19 & 20 & 21 & 22 & 23 & 24 & 25 & 26 \\
\hline Distance(m) & 8.54 & 8.55 & 8.05 & 7.84 & 8.35 & 8.55 & 8.81 & 8.98 & 9.09 & 10.3 & 10.4 & 10.5 & 10.6 \\
\hline
\end{tabular}

As can be seen from Fig. 11, the distance between the left rear wheel of the truck and the left marking line stabilizes at about 11.9 meters firstly and decreases afterward to the minimum value of 7.84 meters and increases at the end. The overall variation ranges from 7.84 meters to 12.35 meters. Taking the left standard line as the reference, the distance of the right lane in truck driving direction ranges from 11.4 meters to 15.2 meters and that of the left lane ranges from 7.6 meters to 11.4 meters, which means the truck has fully entered the left lane as shown in Table 3. In conclusion, the truck drove in the right lane in its direction, and then changed to the left and entered the left lane completely, and finally turned to the right until it stopped.

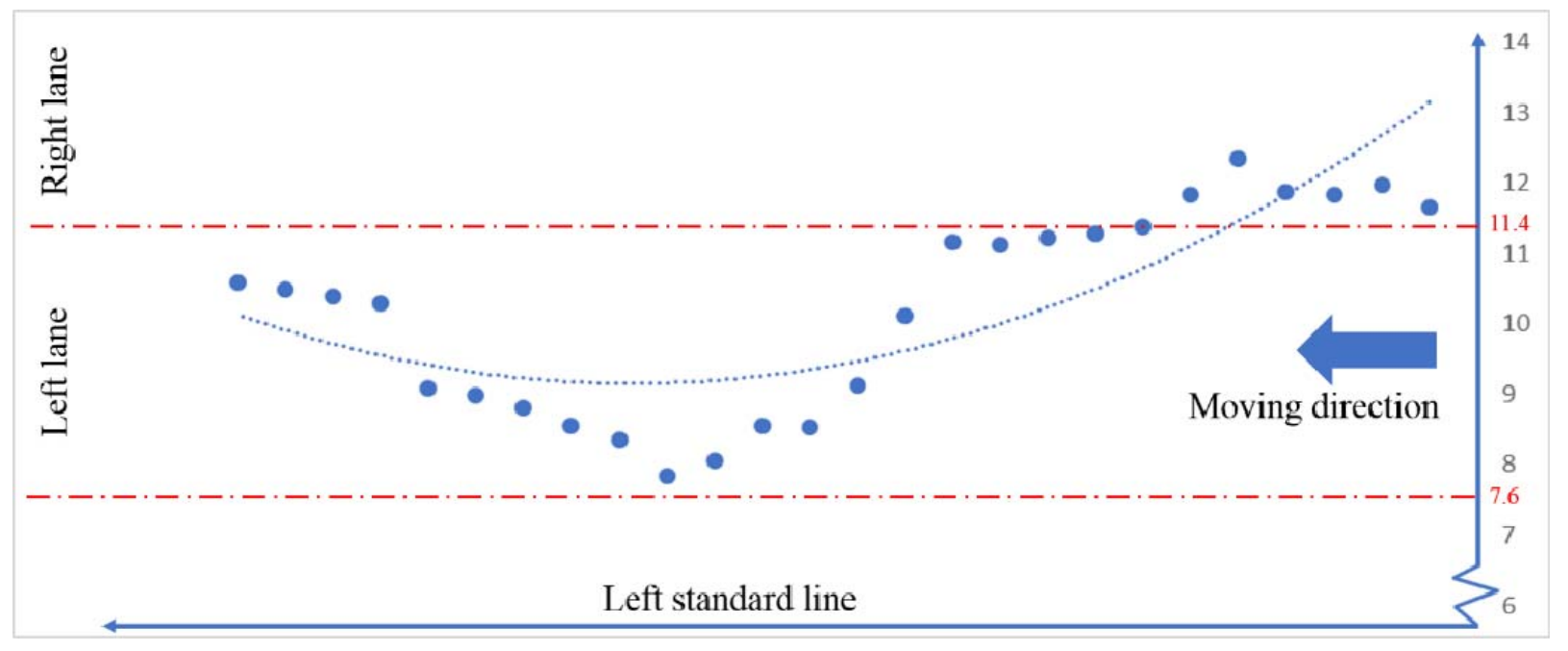

Fig. 11 Top view of truck track point moving trend

\section{Conclusion}

Accurate accident reconstruction is of crucial importance to the traffic regulator in determining the liability of accidents and the researchers to study the safety of vehicles or damage to the human body. However, the location of vehicles or pedestrians usually can't be accurately recorded during traffic accidents. Therefore, computer software such as PC-CRASH and MADYMO is often used in the early stage where the kinetic principle is applied to simulate the process of the accident to meet the final location of the vehicle in the accident. Sometimes the accuracy can't be guaranteed since it is only a compromise that real accident process can't be observed $[7,8]$. With the popularization of urban road surveilling system and driving recorder, the occurrence of a large number of traffic accidents is recorded and saved. Through certain visual measurement techniques and image algorithms, accurate calculation of the positional relationship between target objects and the road surface in traffic accidents with surveillance video can be achieved, and high measurement accuracy can also be guaranteed. Also it should be noted that the lane-dividing lines in the surveillance video presented in this paper are relatively flat, but some other cameras are with wide-angle lenses and the videos taken by them are definitely distorted. Therefore, image distortion must be processed first and that is the further research area to calibrate the surveillance camera and correct the video image. 


\section{Acknowledgements}

This work is financially supported by the National Key Research and Development Program of China (project number: 2016YFC0800702-3) and the authors would like to acknowledge the Chongqing Bayi Traffic Accident Judicial Appraisal Center for providing accident case in this article.

\section{References}

[1]. Babalola O J. Development and pilot study road traffic injury surveillance, KadunaNigeria[J]. Online Journal of Public Health Informatics, 2017, 9(1).

[2]. Chang H, Tsai F. Reconstructing Three-Dimensional Specific Curve Building Models from a Single Perspective View Image[J]. ISPRS - International Archives of the Photogrammetry, Remote Sensing and Spatial Information Sciences, 2012, XXXIX-B6:101-106.

[3]. Yamafune K, Torres R, Castro F. Multi-Image Photogrammetry to Record and Reconstruct Underwater Shipwreck Sites[J]. Journal of Archaeological Method \& Theory, 2016, 23.:1-23.

[4]. Sturm P. Pinhole Camera Model[J]. Computer Vision, 2014:300-321.

[5]. Foroosh H, Cao X, Balci M. Metrology in uncalibrated images given one vanishing point[C]// IEEE International Conference on Image Processing. IEEE, 2014:III-361-4..

[6]. Pall E, Mathe K, Tamas L, et al. Railway track following with the AR.Drone using vanishing point detection[C]// IEEE International Conference on Automation, Quality and Testing, Robotics. IEEE, 2014:1-6.

[7]. Sun J, Chen X, Gong Z, et al. Accurate camera calibration with distortion models using sphere images[J]. Optics \& Laser Technology, 2015, 65(65):83-87.

[8]. Radu A I, Cofaru C, Tolea B, et al. Study regarding seat's rigidity during rear end collisions using a MADYMO occupant model[J]. 2017, 252(1):012004. 